\section{Self-rated health in urban adults, perceptions of the physical and social environment, and reported comorbidities: The BH Health Study}

\author{
Autoavaliação da saúde em adultos urbanos, \\ percepção do ambiente físico e social e relato de \\ comorbidades: Estudo Saúde em Beagá
}

\author{
Autoevaluación de la salud en adultos urbanos, \\ percepción del medio físico y social, y \\ comorbilidad: Estudio Salud en Beagá
}

\begin{abstract}
This study assesses the prevalence of poor selfrated health and investigates its association with individual and environmental characteristics in adults with and without reported morbidity. A household survey assessed 4,048 adults in two districts of Belo Horizonte, Minas Gerais State, Brazil. We used Poisson regression with robust variance stratified by the presence of reported morbidity. Prevalence of poor self-rated health was 29.9\% (42.6\% in those with morbidity and $13.1 \%$ in the group without morbidity). All assessed domains were associated with selfrated health in subjects with reported morbidity. In the group without reported morbidity, the following were associated with self-rated health: social environment, socio-demographic factors, lifestyle, and psychological health. Perceived problems in the environment were associated with poor self-rated health in both groups, even after hierarchical adjustment. The results suggest the importance of investigating self-rated health stratified by reported morbidity and reinforce the need to include variables that characterize the physical and social environment.
\end{abstract}

Health Status; Morbidity; Urban Health

\author{
Adriana Lúcia Meireles 1,2 \\ César Coelho Xavier 2,3 \\ Amanda Cristina de Souza Andrade 1,2 \\ Amélia Augusta de Lima Friche 1,2 \\ Fernando Augusto Proietti 3,4 \\ Waleska Teixeira Caiaffa 1,2
}

\section{Resumo}

Este estudo avalia a prevalência de autoavaliação da saúde ruim e investiga sua associação com características individuais e da percepção do ambiente em indivíduos com e sem o relato de morbidades. Inquérito domiciliar com 4.048 adultos de dois distritos de Belo Horizonte, Minas Gerais, Brasil. Realizou-se análise de regressão de Poisson com variância robusta estratificada pela presença de morbidade referida. Prevalência de autoavaliação da saúde ruim igual a 29,9\%, sendo 42,6\% nos indivíduos com morbidade referida e 13,1\% nos adultos sem morbidade referida. Todos os domínios avaliados foram associados à autoavaliação da saúde nos indivíduos com morbidade referida. No grupo sem morbidade referida, associaram-se à autoavaliação da saúde: ambiente social, sociodemográfico, estilo de vida e saúde psicológica. Problemas percebidos no ambiente de moradia foram associados à autoavaliação da saúde ruim em ambos os grupos, mesmo após ajustamento hierarquizado. Os resultados sugerem a importância da investigação da autoavaliação da saúde de forma estratificada pela presença e ausência de morbidades referidas, e reforçam a necessidade da inclusão de variáveis do ambiente físico e social dos indivíduos.

Nível de Saúde; Morbidade; Saúde Urbana 


\section{Introduction}

Self-rated health has received growing attention in the international literature, since it centers on the assessment of health status and relates to the population's well-being and life satisfaction 1 .

Many studies have aimed to understand the significance of self-rated health, due not only to the measure's robustness, but also the possibility of its use in large population surveys. Self-rated health is a valid, reliable measure of health and strong predictor of morbidity, mortality, and use of health services 2,3 , and is a valuable source of data on the population's health status 4 . It is also simple, inexpensive, and easy to administer in health surveys 2 , including in different populations $5,6,7$.

Individual factors that can influence selfrated health have been extensively studied 1 . Evidence suggests that self-rated health is a multidimensional construct with various domains, including demographics, lifestyle, and psychological and physical health 2,8 , but which can differ according to age and gender 2,4,8,9,10.

In most population studies, physical health tends to be the principal determinant of self-rated health 2,5 . However, few studies have focused on the difference between factors associated with self-rated health in the presence and absence of diseases 2,5,11,12. This perspective becomes relevant when assessing the high prevalence of reported diseases in population studies 13 .

According to Brazil's 2003 National Household Sample Survey (PNAD), 40\% of the country's population 18 or older reported at least one chronic disease among the 12 included in the survey ${ }^{13}$. In the Survey on the Social Dimension of Inequalities (PDSD) conducted in 2008 with 12,324 individuals 20 years or older, which investigated the presence of self-reported comorbidity based on a list of 16 diseases, $63.3 \%$ of participants reported one or more diseases 1 .

Another relevant aspect for understanding possible underlying factors in self-rated health extends beyond individual factors to the dimensions of the physical and social setting. A growing number of studies incorporate environmental characteristics as determinants of self-rated health (although fewer in number and with less intensity when compared to those contemplating individual characteristics) 14 . Two pillars of urban health assist the understanding of the environment's influence on self-rated health: the role of the physical and social setting in shaping individual health and the need to measure phenomena involving unjust and avoidable inequalities in the environment and in health 15 .
Given the above, this study aimed to assess the prevalence of poor self-rated health among adults in a large Brazilian city and to investigate the association between self-rated health and individual characteristics and perception of the environment among those with and without selfreported diseases.

\section{Methods}

Data were obtained from The BH Health Study (Estudo Saúde em Beagá), a population-based household survey conducted by the Belo Horizonte Observatory for Urban Health (OSUBH) from 2008 to 2009 in two of the nine health districts (Barreiro and Oeste) in Belo Horizonte, Minas Gerais State, Brazil. The two districts were selected due to field research logistics and their internal heterogeneity in terms of various demographic, socioeconomic, and health indicators. Details on the survey have been published previously 16,17 .

\section{Sample}

The study used stratified cluster sampling in three stages: census tract; household; and within the household, an adult resident ( $>18$ years of age) and an adolescent 11 to 17 years of age. Participation rate was $80 \%$, resulting in 4,048 interviews with adults ( $>18$ years) and 1,042 with adolescents (11 to 17 years). The adults, the focus of this study, answered face-to-face interviews and had their weight, height, and waist circumference measured.

\section{Data collection instruments}

The BH Health Study used a standardized questionnaire prepared specifically for this study, with classical questions from Brazilian and international epidemiological surveys. All the instruments were pre-coded and pre-tested in a pilot study. Data were collected with a face-toface interview lasting approximately 40 minutes. Anthropometric measurements were also taken: weight, using a portable digital scale, height, using a stadiometer, and waist circumference, with an inelastic tape measure.

\section{Response variable}

The response variable was self-rated health, measured with the question "In general, would you say that your health is: very good, good, fair, poor, or very poor?" The answers were di- 
chotomized as poor self-rated health (fair, poor, very poor) and good self-rated health (very good, good).

\section{Stratification variable}

Reported morbidity has been considered an approximate measure of the information obtained from current clinical examinations and shows good agreement with patient medical records, especially for some selected conditions such as cardiovascular diseases and diabetes mellitus 12 . In the current study, the stratification variable was reported morbidity, measured by the following question: "Has a physician or other health professional said that you have one of the following diseases: high blood pressure, high cholesterol, diabetes, asthma, arthritis, arthrosis, rheumatism, osteoporosis, chronic kidney disease, depression, migraine, epilepsy, tuberculosis, cancer, heart disease, lung disease, chronic digestive tract disease (ulcer/gastritis), or mental illness?" Individuals were combined into two strata: with morbidity (report of at least one disease) and without morbidity (absence of disease).

\section{Explanatory variables}

The explanatory variables were divided into five blocks according to the adapted Cremonese model 18 for self-rated health (Figure 1).

The first (most distal) block, called physical and social environment, included variables that involved perception of the neighborhood. The choice of variables that evaluated perception of the neighborhood environment was based on previous work conducted by researchers from the OSUBH in the same population sample 16,17,19.

Availability of food, ease in walking around the neighborhood, noise, and feeling of belonging to the neighborhood were obtained from the following questions, respectively: "Does your neighborhood have places to buy food within walking distance?" (yes/no); "Is it easy to walk from one place to another?" (yes/no); "Does the noise bother you?" (yes/no); and "Do you feel part of your neighborhood?” (yes/no).

The item public services in the neighborhood was obtained by adding the answers to the following questions: "In your neighborhood, how do you rate: street lighting; street and sidewalk maintenance; public places for sports and recreation; public equipment such as guardhouses, payphones, trash cans, and pedestrian walkways; public transportation (bus, subway); ease in obtaining fresh/good-quality food; policing (on foot, patrol cars, mounted police); traffic; air quality?" The choices for the answers were scored from 1 to 4 (very good to very poor), and the sum of the items was classified according to the median $(<$ 21 - good; $\geq 21$ - poor).

The item pleasant neighborhood was evaluated by the following questions: "Is your neighborhood a pleasant place for young people and teenagers? Is it a pleasant place for children? Does it have trees that make the environment pleasant?" Social disorder was measured by the questions "Does your neighborhood have: abandoned buildings, houses, or warehouses? Garbage or rubble on the streets and sidewalks? Empty lots full of garbage and rubble or with high weeds? People or places in the neighborhood with loud music, people arguing out loud, or partying late into the night? People walking around armed (other than police)? Gunfire?" Trust in neighbors was assessed by: "In your neighborhood, are there persons that you trust in: To leave your house keys if necessary? Lend things? Leave a family member in case of an emergency?" Neighborhood cooperation was assessed by the questions: "Do people in your neighborhood join together to: Intervene in arguments or problems with other neighborhoods; improve neighborhood safety; avoid drug use in public places; avoid dumping garbage in the neighborhood; demand better health services from the authorities; improve the neighborhood's appearance; demand better schools from the authorities?" And safety by the following questions: "Do you feel safe walking around during the day? Do you feel safe walking around at night?" Categorization of the scores for pleasant neighborhood, social disorder, trust in neighbors, neighborhood cooperation, and safety was obtained by the sum of the questions comprising each variable, categorized as "yes" when individuals answered yes to at least one item and "no" when they answered no to all of the questions.

Social contact was evaluated with the questions: "How often to you go to: bars, pubs, nightclubs, or concert halls; festivals or street parties; clubs or recreational associations; soccer fields/ stadiums; movie theaters; shopping malls; theaters; parks/city squares; dance/cultural/sports groups?" Principal components analysis was performed to reduce the number of variables. The score for the first component was divided into tertiles and classified as low, medium, and high social contact.

The second block included variables from the socio-demographic domain: gender, age bracket, schooling (years of school), and work (currently working or ever worked).

Variables in the lifestyle domain comprised the third block and included: consumption of fruits/greens/vegetables, leisure-time physical activity, smoking, and drinking. 
Model for hierarchical entry of factors associated with self-rated health in adults.

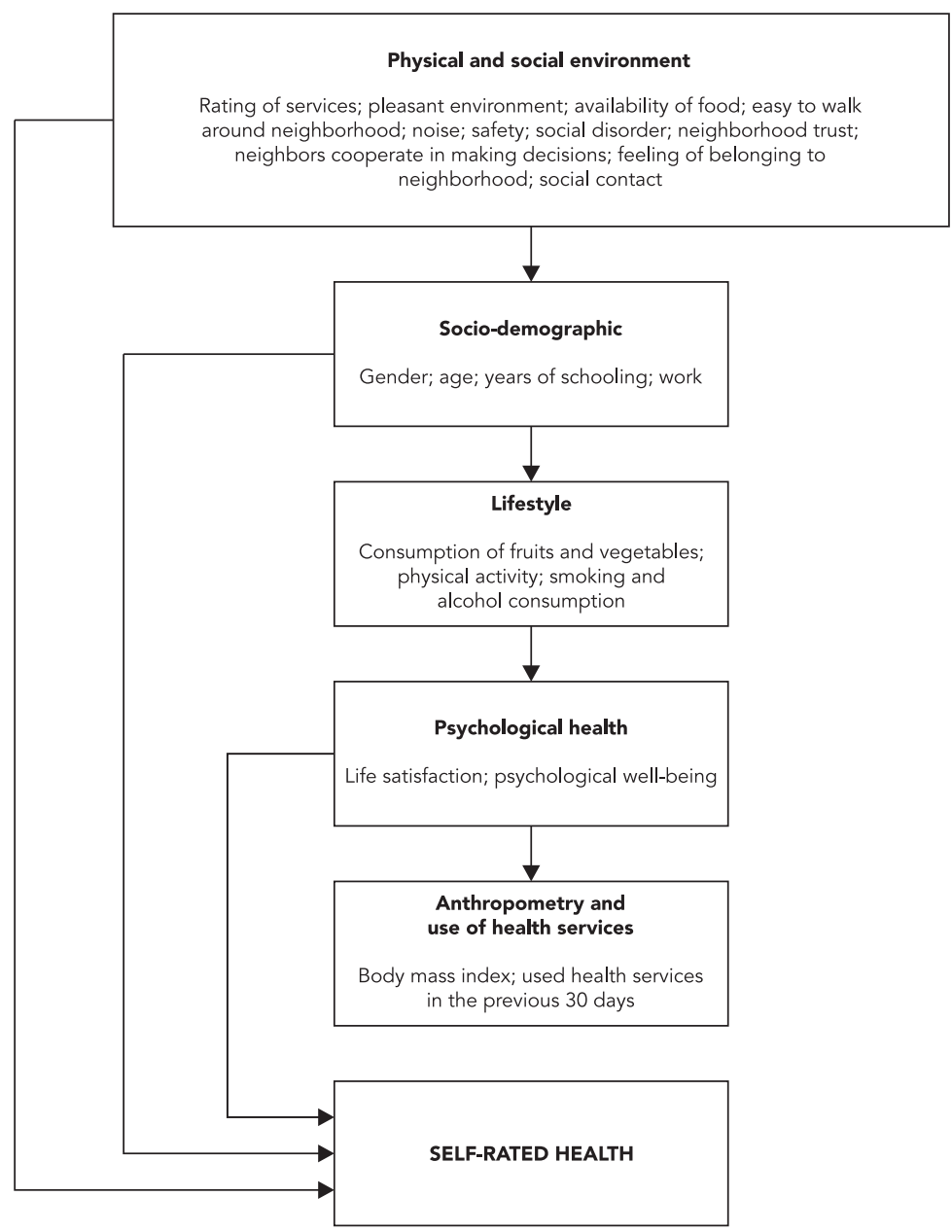

Leisure-time physical activity was measured with the long version of the International Physical Activity Questionnaire (IPAQ), calculating the weekly score for leisure-time physical activity 19 . Individuals were classified below the recommended level with less than 150 minutes of physical activity per week and within the recommended level with greater than or equal to 150 minutes/week 20 .

The fourth block consisted of variables from the psychological health domain: life satisfaction and psychological well-being. The Self-Anchoring Ladder developed by Cantril in 196721 consists of a scale from 1 to 10 , represented schematically by a ladder, in which the lowest rung represents the worst satisfaction with life and the highest rung the best. Individuals choose the rung that they identifed with on the day of the interview. The cutoff for our study was the median for the selected rungs, and the answers were categorized as negative/dissatisfied (rungs 1 to 5 ) and positive/satisfied (rungs 6 to 10).

Psychological well-being was assessed with the Faces Scale developed by Andrews in 1976 21, a schematic instrument consisting of seven faces, representing the person's predominant mood in the two weeks prior to the interview. We opted to categorize the answers as psychological wellbeing (figures 1 to 4 ) and psychological distress (figures 5 to 7), as in a previous study 22 .

Finally, we included variables from anthropometry and use of health services (more proxi- 
mal): body mass index (BMI) and use of health services ("Have you gone to some health service in the last 30 days?”). BMI classification for adults (18 to 59 years) was based on World Health Organization (WHO) guidelines 23: underweight (BMI < 18.50); normal weight (BMI: 18.50-24.99); overweight (BMI: 25.00-29.99); obese (BMI > 29.99). Among the elderly, the study adopted the cutoffs proposed by Lipschtz 24: underweight (BMI < 22.00); normal weight (BMI: 22.00-27.99); overweight (BMI: 27.99-31.99); obese (BMI > 31.99).

\section{Statistical analysis}

Descriptive analyses were performed using frequency distributions, means, medians, and standard deviation (SD). To verify the association between self-rated health and the explanatory variables, we used Poisson regression with robust variance (which provides the best estimates of prevalence ratios (PR) for very frequent outcomes 25) in the bivariate and multivariate analyses, considering presence versus absence of selfreported diseases. Explanatory variables with $\mathrm{p}<$ 0.20 in the bivariate analysis were included in the multivariate analysis.

The analysis adopted hierarchical entry of variables in blocks 26 , with the introduction of more distal to more proximal blocks according to the theoretical model (Figure 1), in the following order: physical and social environment, sociodemographics, lifestyle, psychological health, anthropometry, and use of health services. The order of entry of blocks of variables was based on the theoretical model used by Cremonese et al. 18 . The entry of each block of variables produced a new analytical model. Comparison of the models used the Akaike Information Criterion (AIC).

The analyses incorporated weighting factors that considered design effects of the sample using the SVY command from Stata 12.0 (StataCorp LP, College Station, USA). Significance was set at 5\%, with $95 \%$ confidence intervals $(95 \% \mathrm{CI})$.

The study was approved by the Ethics Research Committee of the Federal University of Minas Gerais (case ETIC 253/06). After a comprehensive explanation and clarification of doubts, study subjects agreed to participate in the survey and signed a free and informed consent form before the interview.

\section{Results}

Prevalence of poor self-rated health in the study sample was $29.9 \%$ (95\%CI: $28.0-31.9 \%$ ). Prevalence of poor self-rated health in the group with reported morbidity was $42.6 \%$ (95\%CI: 40.2 -
$45.0 \%$ ), compared to $13.1 \%$ (95\%CI: $10.9-15.3 \%$ ) in the group without morbidity. Approximately $57 \%$ of the interviewees reported one or more diseases, and as the number of reported diseases increased, self-rated health worsened (1 disease - PR: 2.4 and 95\%CI: 1.65-2.53; 2 diseases - PR: 3.16 and 95\%CI: 2.61-3.82; 3 or more diseases PR: 4.94 and 95\%CI: 4.13-5.90), showing a linear relationship between self-rated health and reported morbidity. The study population included a majority of women $(53.1 \%)$ and individuals from 25 to 59 years of age (68.8\%), with a mean age of 44.9 years $(\mathrm{SD}=16.83)$. As for schooling, $40.8 \%$ had eight years of school or less. Prevalence of poor self-rated health increased with age, independently of gender $(\mathrm{p}<0.001)$. Comparing men and women, significant differences were only found in the 25 to 59 year age bracket $(\mathrm{p}=0.009)$.

Mean age in the group without reported morbidity was 36 years (SD:13.46) and median age was 33.5 years; $31.3 \%$ reported eight years of schooling or less; and $72.8 \%$ were currently working or had worked. Among those with reported morbidity, mean and median age was 49.0 years (SD: 16.92 ); $47.9 \%$ had eight years of schooling or less; and $58.4 \%$ were working or had worked.

Results of the bivariate and multivariate analyses for poor self-rated health are shown according to the stratified analysis for individuals with and without reported comorbidity.

\section{Stratum with one or more reported diseases}

For socio-demographic variables, worse self-rated health was associated with increasing age and less schooling. Currently working or ever having worked was a protective factor against poor self-rated health. In the other domains related to individual characteristics (lifestyle, psychological health, anthropometry, and use of health services), all the variables showed statistical associations with self-rated health (Table 1).

As for the physical and social environment, perception of an unpleasant neighborhood environment, not trusting neighbors, not feeling part of the neighborhood, and low social contact were associated with poor self-rated health among individuals that reported one or more diseases (Table 2).

In the hierarchical multivariate analysis (Table 3), poor physical and social environment and poor self-rated health remained associated with each other. Individuals with poor self-rated health viewed their neighborhood as unpleasant, had low social contact, and distrusted their neighbors when compared to their peers (model 1). 
Table 1

Distribution, prevalence rates, and crude prevalence ratios for poor self-rated health in relation to individual variables. Belo Horizonte, Minas Gerais State, Brazil, 2008-2009.

\begin{tabular}{|c|c|c|c|c|c|c|}
\hline \multirow[t]{2}{*}{ Variables } & \multicolumn{3}{|c|}{ With reported morbidity $(n=2,539)$} & \multicolumn{3}{|c|}{ Without reported morbidity $(n=1,498)$} \\
\hline & $n(\%)$ & $\begin{array}{l}\text { Prevalence of poor } \\
\text { self-rated health }\end{array}$ & PR $(95 \% \mathrm{Cl})$ & $n(\%)$ & $\begin{array}{l}\text { Prevalence of poor } \\
\text { self-rated health }\end{array}$ & PR $(95 \% \mathrm{Cl})$ \\
\hline \multicolumn{7}{|l|}{ Socio-demographic } \\
\hline \multicolumn{7}{|l|}{ Gender } \\
\hline Female & $1,651(59.41)$ & 43.27 & 1.00 & $730(44.62)$ & 15.07 & 1.00 \\
\hline Male & $888(40.59)$ & 41.60 & $0.96(0.85-1.09)$ & $768(55.38)$ & 11.49 & $0.76(0.55-1.06)$ \\
\hline \multicolumn{7}{|l|}{ Age (years) } \\
\hline $18-24$ & $187(9.29)$ & 24.30 & 1.00 & $334(26.70)$ & 9.13 & 1.00 \\
\hline $25-59$ & $1,619(68.37)$ & 41.41 & $1.70(1.24-2.33)$ * & $1,066(69.37)$ & 14.42 & $1.58(1.03-2.42) * *$ \\
\hline$\geq 60$ & $733(22.34)$ & 53.82 & $2.21(1.60-3.06)$ * & 98 (3.92) & 16.49 & $1.81(1.02-3.21)^{* *}$ \\
\hline \multicolumn{7}{|l|}{ Schooling (years) } \\
\hline$\geq 12$ & $400(20.48)$ & 24.51 & 1.00 & 301 (25.39) & 6.08 & 1.00 \\
\hline $9-11$ & $758(31.66)$ & 36.38 & $1.48(1.16-1.90) * \star \star$ & $646(43.28)$ & 10.25 & $1.69(0.93-3.05)$ \\
\hline $5-8$ & $532(21.79)$ & 48.30 & $1.97(1.57-2.47)$ * & $342(20.91)$ & 17.72 & $2.91(1.56-5.46)$ * \\
\hline $0-4$ & $845(26.07)$ & 59.47 & $2.43(1.95-3.02)$ * & $209(10.41)$ & 32.64 & $5.37(2.90-9.94)$ * \\
\hline \multicolumn{7}{|l|}{$\begin{array}{l}\text { Currently working or ever } \\
\text { worked? }\end{array}$} \\
\hline No & $1,207(41.57)$ & 52.14 & 1.00 & $381(27.17)$ & 15.57 & 1.00 \\
\hline Yes & $1,332(58.43)$ & 35.80 & $0.69(0.61-0.77)$ * & $1,117(72.83)$ & 12.16 & $0.78(0.56-1.08)$ \\
\hline \multicolumn{7}{|l|}{ Lifestyles } \\
\hline \multicolumn{7}{|l|}{$\begin{array}{l}\text { Consumption of fruits and } \\
\text { vegetables (days/week) }\end{array}$} \\
\hline$\geq 5$ & $1,367(52.90)$ & 37.78 & 1.00 & $702(44.83)$ & 9.06 & 1.00 \\
\hline$<5$ & $1,172(47.10)$ & 47.99 & $1.27(1.13-1.43)$ *** & $796(55.17)$ & 16.36 & $1.81(1.31-2.49)$ * \\
\hline \multicolumn{7}{|l|}{ Physical activity } \\
\hline Recommended level & $588(26.72)$ & 29.79 & 1.00 & $426(35.05)$ & 6.34 & 1.00 \\
\hline $\begin{array}{l}\text { Below recommended } \\
\text { level }\end{array}$ & $1,803(73.28)$ & 46.85 & $1.57(1.30-1.89)$ * & $988(64.95)$ & 17.63 & $2.78(1.76-4.41) *$ \\
\hline \multicolumn{7}{|l|}{ Smoking } \\
\hline Never & 1,402 (56.09) & 38.22 & 1.00 & $930(64.00)$ & 11.22 & 1.00 \\
\hline Former smoker & $695(26.62)$ & 46.13 & $1.21(1.05-1.39) * \star$ & $277(16.91)$ & 12.36 & $1.10(0.68-1.78)$ \\
\hline Smoker & $442(17.29)$ & 51.33 & $1.34(1.14-1.59) * \star \star$ & $291(19.09)$ & 19.98 & $1.78(1.16-2.73)^{\star \star \star}$ \\
\hline \multicolumn{7}{|l|}{ Alcohol consumption } \\
\hline No & $1,556(54.99)$ & 49.33 & 1.00 & $734(46.74)$ & 14.77 & 1.00 \\
\hline Yes & $983(45.01)$ & 34.35 & $0.69(0.61-0.80)$ * & $764(53.26)$ & 11.61 & $0.79(0.54-1.13)$ \\
\hline \multicolumn{7}{|l|}{ Psychological health } \\
\hline \multicolumn{7}{|l|}{ Psychological well-being } \\
\hline Well-being & $2,170(88.14)$ & 39.35 & 1.00 & $1,367(91.90)$ & 11.65 & 1.00 \\
\hline Distress & $365(11.86)$ & 66.47 & $1.50(1.32-1.70)$ * & $130(8.10)$ & 29.55 & $2.54(1.74-3.69)$ * \\
\hline \multicolumn{7}{|l|}{ Life satisfaction } \\
\hline Satisfied & $1,910(74.63)$ & 37.79 & 1.00 & $1,210(79.10)$ & 10.37 & 1.00 \\
\hline Dissatisfied & $625(25.37)$ & 56.58 & $1.69(1.52-1.88)$ * & $288(20.83)$ & 23.40 & $2.26(1.64-3.10)$ * \\
\hline
\end{tabular}

(continues) 
Table 1 (continued)

\begin{tabular}{|c|c|c|c|c|c|c|}
\hline \multirow[t]{2}{*}{ Variables } & \multicolumn{3}{|c|}{ With reported morbidity $(n=2,539)$} & \multicolumn{3}{|c|}{ Without reported morbidity $(n=1,498)$} \\
\hline & n (\%) & $\begin{array}{l}\text { Prevalence of poor } \\
\text { self-rated health }\end{array}$ & PR $(95 \% \mathrm{Cl})$ & $n(\%)$ & $\begin{array}{l}\text { Prevalence of poor } \\
\text { self-rated health }\end{array}$ & $\mathrm{PR}(95 \% \mathrm{Cl})$ \\
\hline \multicolumn{7}{|c|}{$\begin{array}{l}\text { Anthropometry and use of } \\
\text { health services }\end{array}$} \\
\hline \multicolumn{7}{|l|}{ BMI } \\
\hline Normal & $933(37.00)$ & 35.46 & 1.00 & $816(56.92)$ & 11.48 & 1.00 \\
\hline Underweight & $152(6.18)$ & 38.93 & $1.10(0.82-1.47)$ & $78(5.27)$ & 24.49 & $2.13(1.18-3.84)$ *** \\
\hline Overweight & $1,018(40.23)$ & 46.00 & $1.30(1.11-1.51) * \star \star$ & $422(27.86)$ & 12.05 & $1.05(0.70-1.56)$ \\
\hline Obese & $410(16.60)$ & 52.08 & $1.47(1.24-1.73)$ * & $158(9.95)$ & 20.87 & $1.82(1.11-2.98) * \star \star$ \\
\hline \multicolumn{7}{|c|}{ Use of health service } \\
\hline No & $1,753(69.61)$ & 39.96 & 1.00 & $1,198(80.05)$ & 12.73 & 1.00 \\
\hline Yes & 786 (30.39) & 48.61 & $1.22(1.07-1.38) * \star$ & 300 (19.95) & 14.51 & $1.14(0.73-1.79)$ \\
\hline
\end{tabular}

95\% Cl: 95\% confidence interval; BMI: body mass index; PR: prevalence ratio.

* $p<0.001$;

** $p<0.05$

$\star \star \star ~ p<0.01$

Table 2

Distribution, prevalence rates, and crude prevalence ratios for poor self-rated health and variables from the physical and social environment. Belo Horizonte, Minas Gerais State, Brazil, 2008-2009.

\begin{tabular}{|c|c|c|c|c|c|c|}
\hline \multirow[t]{2}{*}{ Variables } & \multicolumn{3}{|c|}{ With reported morbidity $(n=2,539)$} & \multicolumn{3}{|c|}{ Without reported morbidity $(n=1,498)$} \\
\hline & n (\%) & $\begin{array}{c}\text { Prevalence of } \\
\text { poor self-rated } \\
\text { health }\end{array}$ & PR $(95 \% \mathrm{Cl})$ & n (\%) & $\begin{array}{l}\text { Prevalence of } \\
\text { poor self-rated } \\
\text { health }\end{array}$ & PR $(95 \% \mathrm{Cl})$ \\
\hline \multicolumn{7}{|c|}{ Physical environment } \\
\hline \multicolumn{7}{|c|}{ Rating of public services in neighborhood } \\
\hline Good & $1,154(47.66)$ & 39.77 & 1.00 & $761(53.07)$ & 11.95 & 1.00 \\
\hline Poor & $1,088(52.34)$ & 43.94 & $1.10(0.97-1.25)$ & $624(46.93)$ & 15.43 & $1.29(0.90-1.86)$ \\
\hline \multicolumn{7}{|c|}{ Pleasant neighborhood } \\
\hline Yes & 2,107 (86.99) & 41.35 & 1.00 & $1,280(87.96)$ & 12.97 & 1.00 \\
\hline No & $347(13.01)$ & 52.05 & $1.26(1.05-1.52)$ * & $190(12.04)$ & 14.60 & $1.13(0.72-1.76)$ \\
\hline \multicolumn{7}{|c|}{ Availability of food } \\
\hline Yes & $2,271(87.90)$ & 45.50 & 1.00 & $1,351(89.09)$ & 13.04 & 1.00 \\
\hline No & $267(12.10)$ & 42.20 & $1.08(0.89-1.31)$ & $145(10.91)$ & 13.56 & $1.04(0.66-1.64)$ \\
\hline \multicolumn{7}{|c|}{ Easy to walk around neighborhood } \\
\hline Yes & $2,230(87.09)$ & 41.81 & 1.00 & $1,364(90.20)$ & 12.36 & 1.00 \\
\hline No & 305 (12.91) & 47.39 & $1.13(0.95-1.35)$ & $132(9.80)$ & 20.07 & $1.62(1.05-2.52)$ * \\
\hline \multicolumn{7}{|l|}{ Noise } \\
\hline Yes & $1,172(46.01)$ & 44.69 & 1.00 & 600 (41.09) & 14.37 & 1.00 \\
\hline No & $1,365(53.99)$ & 40.88 & $0.85(0.63-1.16)$ & $896(58.91)$ & 12.21 & $1.18(0.86-1.60)$ \\
\hline \multicolumn{7}{|c|}{ Social environment } \\
\hline \multicolumn{7}{|l|}{ Safety } \\
\hline Yes & $2,457(97.81)$ & 42.66 & 1.00 & $1,475(99.37)$ & 13.03 & 1.00 \\
\hline No & $54(2.19)$ & 46.18 & $1.08(0.73-1.61)$ & $13(0.63)$ & 36.03 & $2.77(1.05-7.32)$ * \\
\hline
\end{tabular}


Table 2 (continued)

\begin{tabular}{|c|c|c|c|c|c|c|}
\hline \multirow[t]{2}{*}{ Variables } & \multicolumn{3}{|c|}{ With reported morbidity $(n=2,539)$} & \multicolumn{3}{|c|}{ Without reported morbidity $(n=1,498)$} \\
\hline & n (\%) & $\begin{array}{c}\text { Prevalence of } \\
\text { poor self-rated } \\
\text { health }\end{array}$ & PR $(95 \% \mathrm{Cl})$ & n (\%) & $\begin{array}{c}\text { Prevalence of } \\
\text { poor self-rated } \\
\text { health }\end{array}$ & PR $(95 \% \mathrm{Cl})$ \\
\hline \multicolumn{7}{|c|}{ Trust in neighborhood } \\
\hline Yes & $2,273(90.29)$ & 41.46 & 1.00 & $1,320(88.63)$ & 13.07 & 1.00 \\
\hline No & $236(9.71)$ & 53.75 & $1.30(1.11-1.51)$ * & $159(11.37)$ & 13.13 & $1.00(0.63-1.60)$ \\
\hline \multicolumn{7}{|c|}{ Neighbors' cooperation } \\
\hline Yes & $2,204(86.86)$ & 45.32 & 1.00 & $190(12.93)$ & 15.30 & 1.00 \\
\hline No & $335(13.14)$ & 42.18 & $0.93(0.78-1.10)$ & $1,308(87.07)$ & 12.76 & $0.83(0.55-1.27)$ \\
\hline \multicolumn{7}{|c|}{ Social disorder } \\
\hline Yes & $2,016(83.96)$ & 43.38 & 1.00 & $1,201(82.62)$ & 13.08 & 1.00 \\
\hline No & $431(16.04)$ & 40.47 & $1.07(0.90-1.27)$ & $254(17.38)$ & 13.98 & $0.94(0.61-1.42)$ \\
\hline \multicolumn{7}{|c|}{ Feeling of belonging to neighborhood } \\
\hline Yes & $2,168(86.92)$ & 41.42 & 1.00 & $245(16.32)$ & 13.22 & 1.00 \\
\hline No & $356(13.08)$ & 49.45 & $1.19(1.02-1.39)$ * & $1,246(83.68)$ & 11.22 & $0.85(0.56-1.29)$ \\
\hline \multicolumn{7}{|c|}{ Social contact } \\
\hline High & $713(33.25)$ & 31.87 & 1.00 & $628(47.75)$ & 7.51 & 1.00 \\
\hline Medium & 824 (33.32) & 38.01 & $1.84(1.54-2.19)$ ** & 485 (30.25) & 13.42 & $1.79(1.19-2.68)$ *** \\
\hline Low & $985(33.43)$ & 58.50 & $1.19(0.98-1.45)$ & 379 (21.99) & 24.99 & $3.33(2.20-5.03) * \star$ \\
\hline
\end{tabular}

95\% Cl: 95\% confidence interval; PR: prevalence ratio.

* $\mathrm{p}<0.05$

${ }^{\star *} p<0.001$;

$\star \star \star p<0.01$

Table 3

Poisson multivariate regression analysis for self-rated health and associated factors in the group with reported morbidity. Belo Horizonte, Minas Gerais State, Brazil, 2008-2009.

\begin{tabular}{|c|c|c|c|c|c|}
\hline \multirow[t]{2}{*}{ Variables } & Model 1 & Model 2 & Model 3 & Model 4 & Model 5 \\
\hline & PR (95\%Cl) & PR (95\%Cl) & PR (95\%Cl) & PR (95\%Cl) & PR (95\%Cl) \\
\hline \multicolumn{6}{|c|}{ Physical and social environment } \\
\hline \multicolumn{6}{|c|}{ Pleasant neighborhood } \\
\hline Yes & 1.00 & 1.0 & 1.00 & 1.00 & 1.00 \\
\hline No & $1.22(1.03-1.46)$ * & $1.32(1.12-1.56) * *$ & $1.27(1.08-1.49) * \star$ & $1.20(1.02-1.41)^{\star}$ & $1.19(1.01-1.41)$ * \\
\hline \multicolumn{6}{|c|}{ Social contact } \\
\hline High & 1.00 & 1.00 & 1.00 & 1.00 & 1.00 \\
\hline Medium & $1.17(0.96-1.42)$ & $0.99(0.82-1.21)$ & $0.96(0.79-1.17)$ & $0.98(0.80-1.20)$ & $0.98(0.80-1.20)$ \\
\hline Low & $1.81(1.52-2.15)$ *** & $1.29(1.07-1.55)$ ** & $1.19(0.98-1.43)$ & $1.18(0.98-1.42)$ & $1.19(0.99-1.43)$ \\
\hline \multicolumn{6}{|c|}{ Trust in neighbors } \\
\hline Yes & 1.00 & 1.00 & 1.00 & 1.00 & 1.00 \\
\hline No & $1.24(1.06-1.44) *$ & $1.22(1.04-1.44)$ * & $1.21(1.02-1.42)$ * & $1.15(0.98-1.34)$ & $1.12(0.96-1.31)$ \\
\hline \multicolumn{6}{|c|}{ Socio-demographic } \\
\hline \multicolumn{6}{|l|}{ Age (years) } \\
\hline $18-24$ & & 1.00 & 1.00 & 1.00 & 1.00 \\
\hline $25-59$ & & $1.48(1.06-2.07)$ * & $1.41(1.00-1.97)$ & $1.40(1.00-1.94)$ & $1.20(0.86-1.68)$ \\
\hline$\geq 60$ & & $1.55(1.09-2.22)$ * & $1.55(1.09-2.21)$ * & $1.59(1.13-2.24)$ ** & $1.45(1.02-2.06)$ * \\
\hline
\end{tabular}

(continues) 
Table 3 (continued)

\begin{tabular}{|c|c|c|c|c|c|}
\hline Variables & $\begin{array}{c}\text { Model } 1 \\
\text { PR }(95 \% \mathrm{Cl})\end{array}$ & $\begin{array}{c}\text { Model } 2 \\
\text { PR }(95 \% \mathrm{Cl})\end{array}$ & $\begin{array}{c}\text { Model } 3 \\
\text { PR (95\%Cl) }\end{array}$ & $\begin{array}{c}\text { Model } 4 \\
\text { PR }(95 \% \mathrm{Cl})\end{array}$ & $\begin{array}{c}\text { Model } 5 \\
\text { PR }(95 \% \mathrm{Cl})\end{array}$ \\
\hline \multicolumn{6}{|l|}{ Gender } \\
\hline Female & & 1.00 & 1.00 & 1.00 & 1.00 \\
\hline Male & & $1.03(0.91-1.17)$ & $1.03(0.91-1.04)$ & $1.05(0.92-1.20)$ & $1.06(0.93-1.22)$ \\
\hline \multicolumn{6}{|l|}{ Schooling (years) } \\
\hline$\geq 12$ & & 1.00 & 1.00 & 1.00 & 1.00 \\
\hline $9-11$ & & $1.48(1.12-1.94) * \star$ & $1.49(1.12-1.97) * \star$ & $1.45(1.09-1.92) * \star$ & $1.45(1.10-1.92) * \star \star$ \\
\hline $5-8$ & & 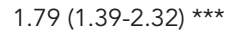 & $1.73(1.32-2.27)^{\star \star \star}$ & $1.67(1.28-2.18) * \star \star$ & $1.64(1.26-2.13)$ *** \\
\hline $0-4$ & & $1.90(1.50-2.42) * \star \star$ & 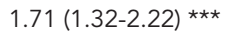 & 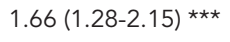 & $1.66(1.28-2.14)$ *** \\
\hline \multicolumn{6}{|c|}{ Currently working or ever worked } \\
\hline No & & 1.00 & 1.00 & 1.00 & 1.00 \\
\hline Yes & & $0.80(0.71-0.91) * *$ & $0.80(0.72-0.91) * \star$ & $0.82(0.72-0.93) * \star$ & $0.82(0.73-0.94) * \star$ \\
\hline \multicolumn{6}{|l|}{ Lifestyles } \\
\hline \multicolumn{6}{|c|}{ Consumption of fruits and vegetables } \\
\hline$\geq 5$ & & & 1.00 & 1.00 & 1.00 \\
\hline$<5$ & & & $1.23(1.09-1.38) * \star$ & $1.20(1.07-1.36) * \star$ & $1.20(1.07-1.35) * \star$ \\
\hline \multicolumn{6}{|l|}{ Physical activity } \\
\hline Recommend level & & & 1.00 & 1.00 & 1.00 \\
\hline Below recommend level & & & $1.24(1.03-1.50)$ * & $1.22(1.01-1.42)$ * & $1.22(1.01-1.47)$ * \\
\hline \multicolumn{6}{|l|}{ Smoking } \\
\hline Never smoked & & & 1.00 & 1.00 & 1.00 \\
\hline Former smoker & & & $1.15(1.01-1.31)$ * & $1.13(0.99-1.29)$ & $1.14(0.99-1.30)$ \\
\hline Smoker & & & $1.29(1.11-1.52) * \star$ & $1.20(1.02-1.42)$ * & $1.28(1.10-1.50) * \star$ \\
\hline \multicolumn{6}{|l|}{ Alcohol consumption } \\
\hline No & & & 1.00 & 1.00 & 1.00 \\
\hline Yes & & & $0.75(0.65-0.88) * \star \star$ & $0.77(0.66-0.89) * \star \star$ & $0.77(0.66-0.89) * * *$ \\
\hline \multicolumn{6}{|l|}{ Psychological health } \\
\hline \multicolumn{6}{|l|}{ Life satisfaction } \\
\hline Satisfied & & & & 1.00 & 1.00 \\
\hline Dissatisfied & & & & $1.29(1.14-1.45) * \star \star$ & $1.29(1.14-1.45) * \star *$ \\
\hline \multicolumn{6}{|l|}{ Psychological well-being } \\
\hline Well-being & & & & 1.00 & 1.00 \\
\hline Distress & & & & 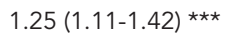 & $1.22(1.08-1.38) * \star$ \\
\hline \multicolumn{6}{|c|}{ Anthropometry and use of health services } \\
\hline \multicolumn{6}{|l|}{$\mathrm{BMI}$} \\
\hline Normal & & & & & 1.00 \\
\hline Underweight & & & & & $0.88(0.66-1.16)$ \\
\hline Overweight & & & & & $1.21(1.05-1.40)$ ** \\
\hline Obese & & & & & $1.41(1.19-1.66)^{* * \star}$ \\
\hline \multicolumn{6}{|l|}{ Use of health services } \\
\hline No & & & & & 1.00 \\
\hline Yes & & & & & $1.18(1.05-1.32)$ ** \\
\hline Akaike Information Criterion & 3894.17 & 3832.37 & 3607.92 & 3564.48 & 3529.96 \\
\hline
\end{tabular}

95\% Cl: 95\% confidence interval; BMI: body mass index; PR: prevalence ratio.

Model 1: adjusted for variables from the physical and social block; Model 2: adjusted for variables from the physical and social environment and sociodemographic blocks; Model 3: adjusted for variables from the physical and social environment, socio-demographic, and lifestyle blocks; Model 4: adjusted for variables from the physical and social environment, socio-demographic, lifestyle, and psychological health blocks; Model 5: adjusted for variables from the physical and social environment, socio-demographic, lifestyle, psychological health, and anthropometry health services use blocks.

* $p<0.05$

** $p<0.01$

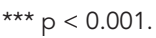


In model 2, poor self-rated health was associated with increasing age, less schooling, and not having worked in life. In model 3, all the variables in the lifestyle block were associated with self-rated health. In model 4 , life dissatisfaction and psychological distress were significantly associated with poor self-rated health, as was the inclusion of the more proximal variables overweight/obesity and recent use of health services (model 5).

In this stratum, even hierarchically organized, rating one's neighborhood as unpleasant remained associated with poor self-rated health in model 5. The multivariate model for individuals with reported morbidity was also similar to that of the total population in terms of the various hierarchical levels analyzed (data not shown).

\section{Stratum without reported morbidity}

A direct relationship was seen between poor self-rated health and age, and an inverse relationship with schooling (Table 1). The following were associated with poor self-rated health: low consumption of fruits/greens/vegetables, low physical activity, current or former smoking, psychological distress, life dissatisfaction, and overweight/obesity (Table 1).

As for physical and social environment, difficulty in walking around the neighborhood, feeling unsafe, and low social contact were significantly associated with poor self-rated health in the bivariate analysis (Table 2).

In the hierarchical multivariate analysis (Table 4), poor self-rated health was associated with low social contact in model 1 ; less schooling in model 2; and low consumption of fruits/greens/ vegetables, physical inactivity, and smoking in model 3 (from the lifestyle block). Life dissatisfaction and psychological distress also showed independent significant association with poor self-rated health in individuals without reported morbidity (model 4). As for the more proximal domain, none of the variables was associated with poor self-rated health when compared to individuals with better self-rated health (model 5).

\section{Discussion}

This study focused on how self-rated health in population studies is modulated by the presence of diseases, producing relevant variations in typically high prevalence rates for worse self-rated health in the subgroup with reported morbidity. Perceived problems in the living environment were associated with poor self-rated health in both study strata, even after hierarchical adjust- ment. The results suggest the importance of investigating self-rated health from a stratified approach based on the presence or absence of reported diseases, contributing to knowledge on self-rated health in population studies and reinforcing the need to include variables that capture individual perception of the physical and social setting.

Overall prevalence of poor self-rated health (29.9\%) was lower than that of residents in Brazilian State capitals based on the Telephone Surveillance System for Risk and Protective Factors for Chronic Diseases (VIGITEL) from 2006 6, which included 54,369 interviewees 18 years or older and used only four response categories (excellent, good, fair, and poor). Combining the fair and poor categories, prevalence of poor self-rated health was $35.3 \%$. The rate was also lower than in the PDSD survey (approximately 40\%) 1, conducted in five geographic regions of Brazil in 2008, with individuals 20 years or older. However, the results show higher prevalence than in the Longitudinal Health Study in Adults (ELSA-Brasil), which assessed 15,105 Brazilian university employees 35 to 74 years of age from 2008 to 2010 and found $19.9 \%$ prevalence of poor self-rated health 27 . A systematic literature review showed greater than $25 \%$ prevalence of poor self-rated health in elderly Brazilians in studies published in recent years 28 .

This disparity could be partially explained by the absence of internationally standardized answers and their categorization 27,28 . Other issues may also explain the different prevalence rates: presence of diseases in the study groups, age of the study groups, information sources (selected interviewees versus third parties), different positioning of questions on the questionnaire, study design issues, and adjustments in the analysis. According to Barros et al. 6 , differences in prevalence rates between studies, countries, and subpopulations may not be consistent with objective health indicators, and may be more attributable to methodological differences, thus hindering comparison 29.

Even after adjustment, the association between poor self-rated health and variables from the neighborhood environment confirm previous results 14,27 , thus reinforcing the importance of incorporating factors from the living environment into self-rated health, based on the presence of diseases in population studies 16 .

Focusing on variables from the environment in each stratum, we see that among individuals with reported morbidity, the variables associated with poor self-rated health were more comprehensive, including physical characteristics (unpleasant neighborhood) and social ones (limited 
Table 4

Poisson multivariate regression analysis for self-rated health and associated factors in the group without reported morbidity. Belo Horizonte, Minas Gerais State, Brazil, 2008-2009.

\begin{tabular}{|c|c|c|c|c|c|}
\hline Variables & $\begin{array}{c}\text { Model } 1 \\
\text { PR }(95 \% \mathrm{Cl})\end{array}$ & $\begin{array}{c}\text { Model } 2 \\
\text { PR }(95 \% \mathrm{Cl})\end{array}$ & $\begin{array}{c}\text { Model } 3 \\
\text { PR }(95 \% \mathrm{Cl})\end{array}$ & $\begin{array}{c}\text { Model } 4 \\
\text { PR }(95 \% \mathrm{Cl})\end{array}$ & $\begin{array}{c}\text { Model } 5 \\
\text { PR }(95 \% \mathrm{Cl})\end{array}$ \\
\hline \multicolumn{6}{|l|}{ Physical and social environment } \\
\hline \multicolumn{6}{|l|}{ Social contact } \\
\hline High & 1.00 & 1.00 & 1.00 & 1.00 & 1.00 \\
\hline Medium & $1.79(1.19-2.68)$ * & $1.43(0.94-2.16)$ & $1.47(0.97-2.23)$ & $1.36(0.91-2.05)$ & $1.36(0.92-2.02)$ \\
\hline Low & $3.33(2.20-5.03)$ ** & $2.05(1.29-3.25)$ * & $1.97(1.25-3.10)$ * & $1.72(1.09-2.73)$ * & $1.72(1.10-2.70)$ * \\
\hline \multicolumn{6}{|l|}{ Socio-demographic } \\
\hline \multicolumn{6}{|l|}{ Age (years) } \\
\hline $18-24$ & & 1.00 & 1.00 & 1.00 & 1.00 \\
\hline $25-59$ & & $1.08(0.69-1.68)$ & $1.00(0.65-1.56)$ & $1.11(0.71-1.73)$ & $1.09(0.71-1.67)$ \\
\hline$\geq 60$ & & $0.77(0.40-1.47)$ & $0.83(0.43-1.64)$ & $0.98(0.49-1.94)$ & $0.93(0.46-1.91)$ \\
\hline \multicolumn{6}{|l|}{ Gender } \\
\hline Female & & 1.00 & 1.00 & 1.00 & 1.00 \\
\hline Male & & $0.78(0.56-1.08)$ & $0.75(0.55-1.03)$ & $0.79(0.58-1.09)$ & $0.78(0.56-1.07)$ \\
\hline \multicolumn{6}{|l|}{ Schooling (years) } \\
\hline$\geq 12$ & & 1.00 & 1.00 & 1.00 & 1.00 \\
\hline $9-11$ & & $1.49(0.83-2.66)$ & $1.33(0.74-2.37)$ & $1.33(0.74-2.38)$ & $1.34(0.75-2.40)$ \\
\hline $5-8$ & & $2.16(1.15-4.06) * \star \star$ & $1.71(0.92-3.17)$ & $1.63(0.88-3.00)$ & $1.59(0.86-2.93)$ \\
\hline $0-4$ & & $3.66(1.94-6.92) * \star$ & $2.77(1.44-5.34)$ * & $2.70(1.44-5.09)$ * & $2.56(1.35-4.85)$ * \\
\hline \multicolumn{6}{|l|}{ Lifestyles } \\
\hline \multicolumn{6}{|l|}{$\begin{array}{l}\text { Consumption of fruits and } \\
\text { vegetables (days/week) }\end{array}$} \\
\hline$\geq 5$ & & & 1.00 & 1.00 & 1.00 \\
\hline$<5$ & & & $1.39(1.03-1.90) * \star \star$ & $1.27(0.94-1.72)$ & $1.28(0.95-1.73)$ \\
\hline \multicolumn{6}{|l|}{ Physical activity } \\
\hline Recommended level & & & 1.00 & 1.00 & 1.00 \\
\hline Below recommended level & & & $1.64(1.03-2.60) * \star \star$ & $1.57(0.99-2.51)$ & $1.53(0.97-2.41)$ \\
\hline \multicolumn{6}{|l|}{ Smoking } \\
\hline Never smoked & & & 1.00 & 1.00 & 1.00 \\
\hline Former smoker & & & $0.99(0.61-1.60)$ & $0.99(0.62-1.59)$ & $0.97(0.61-1.54)$ \\
\hline Smoker & & & 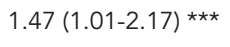 & $1.42(0.97-2.06)$ & $1.42(0.97-2.08)$ \\
\hline \multicolumn{6}{|l|}{ Psychological health } \\
\hline \multicolumn{6}{|l|}{ Life satisfaction } \\
\hline Satisfied & & & & 1.00 & 1.00 \\
\hline Dissatisfied & & & & $1.75(1.26-2.44)$ * & $1.77(1.26-2.47)$ * \\
\hline \multicolumn{6}{|l|}{ Psychological well-being } \\
\hline Well-being & & & & 1.00 & 1.00 \\
\hline Distress & & & & $1.64(1.11-2.42)$ * & $1.63(1.11-2.40)$ * \\
\hline
\end{tabular}

(continues) 
Table 4 (continued)

\begin{tabular}{|c|c|c|c|c|c|}
\hline Variables & $\begin{array}{c}\text { Model } 1 \\
\text { PR }(95 \% \mathrm{Cl})\end{array}$ & $\begin{array}{c}\text { Model } 2 \\
\text { PR }(95 \% \mathrm{Cl})\end{array}$ & $\begin{array}{c}\text { Model } 3 \\
\text { PR }(95 \% \mathrm{Cl})\end{array}$ & $\begin{array}{c}\text { Model } 4 \\
\text { PR }(95 \% \mathrm{Cl})\end{array}$ & $\begin{array}{c}\text { Model } 5 \\
\text { PR }(95 \% \mathrm{Cl})\end{array}$ \\
\hline \multicolumn{6}{|c|}{ Anthropometry and use of health } \\
\hline \multicolumn{6}{|c|}{ services } \\
\hline \multicolumn{6}{|l|}{ BMI } \\
\hline Normal & & & & & 1.00 \\
\hline Underweight & & & & & $1.59(0.94-2.70)$ \\
\hline Overweight & & & & & $1.08(0.73-1.59)$ \\
\hline Obese & & & & & $1.53(0.90-2.57)$ \\
\hline Akaike Information Criterion & 1214.53 & 1204.55 & 1158.52 & 1144.57 & 1126.35 \\
\hline
\end{tabular}

95\% Cl: 95\% confidence interval; BMI: body mass index; PR: prevalence ratio.

Model 1: adjusted for variables from the physical and social block; Model 2: adjusted for variables from the physical and social environment and sociodemographic blocks; Model 3: adjusted for variables from the physical and social environment, socio-demographic, and lifestyle blocks; Model 4: adjusted for variables from the physical and social environment, socio-demographic, lifestyle, and psychological health blocks; Model 5: adjusted for variables from the physical and social environment, socio-demographic, lifestyle, psychological health, and anthropometry health services use blocks.

$\star p<0.01$;

$\star * p<0.001$;

$\star \star \star p<0.05$.

social contact and not trusting neighbors). In the group without reported morbidity, only low social contact was associated with poor self-rated health. Social contact relates to how individuals incorporate social activities into their daily lives, including religious groups, volunteering, political groups, and sports or recreational groups, and is considered one of the main components of social capital 30 . In fact, studies have suggested the association between self-rated health and the individual's level of social contact, indicating that this construct may be a benefit of maintaining good health, providing emotional support, personal fulfillment, and access to information on healthy lifestyles, besides protecting against the negative effects of social isolation ${ }^{30}$. Social activities can modulate individuals' daily lives, facilitating healthy choices and encouraging them to avoid risky behaviors 30 .

In the current study, social contact assessed interviewees' participation in community and leisure-time activities, which the international literature has viewed as part of what authors call social participation. Meanwhile, social and civic participation is a component of social capital (the target of much debate and public policies), and participation can vary widely, from social groups to activities that focus more on civic issues 31 .

Baum et al. 31 assessed the level of social and civic participation in Australia and showed that exclusion from activities is associated with worse health. The authors studied different types of social participation, one of which (similar to our study) they called "social contact through activities in public spaces” (p. 417).

Another variable related to social capital and associated with self-rated health in the group with reported morbidity was trust in neighbors. Studies have shown that social participation, development of trust, and social networks form the basis of social capital 31,32 and that social capital, measured at the individual or contextual level, is directly associated with the population's mental and physical health 32 .

Importantly, social contact and trust between persons and groups are essential for health promotion and are part of the conceptual model for Urban Health, which considers the relevant influence of social networks on the urban population's health 15.

Another highlight of the theoretical model and the results, beyond the variables from the physical and social environment, was the role of psychological health in self-rated health. Life dissatisfaction and/or psychological distress were significantly related to poor self-rated health, regardless of the presence or absence of reported diseases, even after adjusting for hierarchically higher levels. This result is unprecedented according to our literature review, since most studies have failed to investigate psychological factors, while many were limited to sociodemographic factors and risk and health-related behaviors 1,13 .

This association leads us to hypothesize (especially for the group with reported morbidity) that when individuals stay inside longer due to 
illness, the neighborhood environment and relations with neighbors could be relevant and thereby influence self-rated health, perhaps mediated by markers from the psychological health domain.

Considering that psychological factors have been associated with other health outcomes 5,33 , this finding suggests that investigation of the causes, prevention, and management of emotional or psychological problems should be a priority for health services 33 . Therefore, psychological health indicators once again suggest that self-rated health goes beyond physical health, highlighting its multidimensional nature and relationship to physical, mental, and social well-being.

In relation to the other results, the literature corroborates the associations found here. Different studies have shown that increasing age and less schooling correlate with poor self-rated health, justified by the argument that aging is generally accompanied by worsening overall health status, as a function of the increase in diseases and functional incapacities 1,7. Among individuals that report having diseases, currently working or having worked some time in life proved to be a protective factor against poor self-rated health. The association between negative self-rated health and lack of an occupational activity has been shown in previous studies, with special emphasis on those assessing socio-demographic determinants of self-rated health in Brazilian adults 6 .

Lifestyles appeared as important factors associated with poor self-rated health. Previous Brazilian population studies have found this association with behaviors and habits, such as low consumption of fruits and vegetables, lack of physical exercise, and alcohol consumption both in adults 6 and the elderly ${ }^{7}$.

Physical health, ideally evaluated by objective health measures, has been considered the principal determinant of self-rated health 9. Proximal factors for self-rated health according to the current study included BMI (measured during the survey) and reported use of health services. When adjusted for the more distal hierarchical levels, these variables only remained associated with poor self-rated health in the group with diseases. Individuals with excess weight (overweight and obesity) had worse self-rated health in this stratum. The literature shows the association between excess weight and worse self-rated health in the adult and elderly population 2,7 . Borim et al. 7 highlight that since excess weight is related to the presence of chronic diseases, functional incapacity, and problems with social interaction, it should be the focus of attention for everyone working in public health, emphasizing the importance of weight control to improve individual quality of life and well-being. Since excess weight is considered one of the principal modifiable risk factors in the development of chronic noncommunicable diseases (CNCDs), it has been the target of various public policies to prevent and control CNCDs 34 .

Further in relation to the group with reported morbidity, individuals that had used health services in the previous 30 days showed higher odds of reporting worse self-rated health. Poor self-rated health has been associated with increased use of health services 1,5 . Since the search for health care reflects individual perceived need, understanding self-rated health and the profile of individuals with worse self-rated health is essential for organizing health services.

According to our proposed conceptual model for hierarchical analysis, in the group without comorbidity the most proximal domain (represented by anthropometry and use of health services) did not remain associated with self-rated health when adjusted for the more distal hierarchical levels.

Some potential limitations should be considered, including the cross-sectional nature of the data (making it impossible to establish temporal relations) and the option to use self-reporting for health, illness, and perception of the environment. This type of study can involve a survival bias, since the healthiest individuals are the ones that survive the longest, potentially leading to over or underestimation of the real magnitude of poor self-rated health in the population.

The choice to use self-rated health as the response variable was based on its recognition as a valid indicator and strong predictor of mortality that correlates highly with objective health measurements 2,3,6. However, some authors have questioned the use of this indicator for international comparisons and those between subpopulations, arguing that different definitions of health are influenced by cultural and social factors and that the results are not always consistent with objective health indicators 6 . Considering that objective measurements of health such as clinical examinations and laboratory tests are relevant in the attempt to control for potential confounders 35,36, and that The BH Health Study only objectively measured the participants' weight, height, and waist circumference, a more in-depth analysis is assumed to be impossible, thus representing a limitation for this study and for many other studies on this theme published in the literature.

Reported morbidity can be influenced by access to health services and by recall bias. Again, 
based on the widespread use of reported morbidity in health surveys (justified by the high cost and operational difficulties in objectively measuring the presence of diseases, in addition to the good results with the technique's validity, as demonstrated by the its good sensitivity and specificity for detecting health conditions), we believe that it poses only a minor limitation to the study 12,36 .

For physical and social environment, subjective measurements were also used, based on individual perception, knowledge, and/or valuation concerning a given characteristic of the environment. That is, the current study did not include objective measurements of the environment that can be obtained from systematic observation of the environment or geoprocessed data. Thus, interpretation of the results should consider the possibility of a "common source" bias 37 , since individuals' perception of the environment can be influenced by personal factors, besides the fact that individuals' choice of living place may be based on their health or their predisposition to given behaviors.

Another aspect that should be considered is the age difference between the two study strata. As expected, the group with reported comorbidity was older, reflecting population aging and naturally the resulting presence of chronic diseases in the population. However, the doseresponse gradient observed in both strata (even after adjusting for age within each stratum) and the maintenance of associations in the higher hierarchical levels suggest that perception of the environment suffered little impact from age on self-rated health.

Importantly, factors associated with selfrated health in individuals that reported diseases were very similar to those in the total study population (results not shown), which could be explained by the higher prevalence of individuals with reported morbidity $(57.1 \%)$ in the overall population. Considering that the mean age of the study population in The BH Health Study did not differ from that of the total population of Belo Horizonte (Instituto Brasileiro de Geografia e Estatística; http://www.censo2010.ibge. gov.br), what actually appears is an urban population suffering the impacts of aging, and thus highly influenced by the presence of comorbid- ity, thereby justifying the stratification adopted in this study. This can be considered in public policy recommendations for health promotion and prevention that target the adult population and specifically the 25 to 59 year age bracket, which increasingly suffers the impacts of chronic diseases.

The hierarchical analytical model was chosen to understand the determinants of self-rated health, because this type of analysis is not based exclusively on statistical significance and considering the hierarchical conceptual structure, involving various levels (distal, intermediate, and proximal) 24 . However, future studies should consider the use of multilevel analyses capable of dealing with the effects of possible clusters among the characteristics of individuals and their contexts, leading to a more refined measurement of the contribution by each level (individual and contextual) to the determinants of self-rated health 14 .

Since the study was designed to be conducted in two of the city's nine health districts, it did not aim to represent the city's entire adult population, but rather the city's variability in terms of some socioeconomic indicators and health vulnerability - proxies for the population's health inequalities. Therefore, generalization of the data from the two health districts should be done with caution.

Thus, even considering its limitations, the current study provides relevant information for the scientific literature while contributing to the development of future surveys and policies and actions aimed at the population's health promotion. The results emphasize that self-rated health is a multidimensional construct, influenced by individual and environmental factors and suggesting that future studies using self-rated health as a health indicator should distinguish between persons with and without morbidity in the study population, in addition to including variables that characterize the physical and social environment, especially the latter - one of the objects of the current study. We further highlight the importance of including variables that mark psychological health as a determinant of self-rated health, especially in younger populations with lower prevalence of health problems. 


\section{Resumen}

Este estudio evalúa la prevalencia de una peor autopercepción de salud e investiga su relación con las características individuales y ambientales en adultos con y sin morbilidad. Se realizó una encuesta en hogares con 4.048 adultos de dos distritos de Belo Horizonte, Minas Gerais, Brasil. Se utilizó la regresión de Poisson con varianza robusta estratificada por la presencia de morbilidad. La prevalencia de peor autopercepción de salud fue de un 29,9\%; en aquellos con morbilidad fue de un 42,6\%; en el estrato sin morbilidad fue de un 13,1\%. Todos los dominios evaluados se asociaron con la autopercepción de salud en pacientes con morbilidad. En el grupo sin la morbilidad, se asociaron con la autopercepción de salud: el entorno social, sociodemográfico, estilos de vida y la salud psicológica. Los problemas que se observan en el entorno del hogar se asociaron con una mala autopercepción de salud en ambos grupos, incluso después de un ajuste jerárquico. Este estudio sugiere la importancia del análisis de los factores asociados con la autopercepción de salud estratificados por morbilidad y refuerza la necesidad de la inclusión de variables que caractericen el ambiente.

Estado de Salud; Morbilidad; Salud Urbana

\section{Contributors}

A. L. Meireles participated in the data collection, entry, and analysis and worked on the literature review, theoretical and analytical conceptualization, and elaboration of the article. C. C. Xavier worked on the data collection, theoretical discussion of the text, and writing of the article. A. C. S. Andrade worked on the data analysis, theoretical and analytical discussion of the text, and writing of the article. A. A. L. Friche worked on the data collection, theoretical conceptualization, and final version of the article. F. A. Proietti participated in the data collection and final version. W. T. Caiaffa worked on the data collection, conceptualization of the study, theoretical and analytical discussion, and elaboration of the article.

\section{Acknowledgments}

The authors wish to thank the members and coordinators of the Belo Horizonte Observatory for Urban Health who participated in The BH Health Study and the Belo Horizonte Municipal Health Department for its support. For funding The BH Health Study, the authors thank the National Health Fund of the Brazilian Ministry of Health (grant 25000.102984/2006-97), CNPq (grants 475004/2006-0 and 409688/2006-1), and the Minas Gerais State Research Foundation, FAPEMIG (grant APQ-00975-08). W. T. Caiaffa and F. A. Proietti received research grants from $\mathrm{CNPq}$.

\section{References}

1. Pavão ALB, Werneck GL, Campos MR. Autoavaliação do estado de saúde e a associação com fatores sociodemográficos, hábitos de vida e morbidade na população: um inquérito nacional. Cad Saúde Pública 2013; 29:723-34.

2. Molarius A, Janson S. Self-rated health, chronic diseases, and symptoms among middle-aged and elderly men and women. J Clin Epidemiol 2002; 55:364-70.

3. Lima-Costa MF, Cesar CC, Chor D, Proietti FA. Selfrated health compared with objectively measured health status as a tool for mortality risk screening in older adults: 10-Year Follow-up of the Bambuí Cohort Study of Aging. Am J Epidemiol 2012; 175:228-35.

4. McFadden E, Luben R, Bingham S, Wareham N, Kinmonth A, Khaw K. Social inequalities in selfrated health by age: cross-sectional study of 22457 middle-aged men and women. BMC Public Health 2008; 8:230.
5. Cott CA, Gignac MAM, Badley EM. Determinants of self rated health for Canadians with chronic disease and disability. J Epidemiol Community Health 1999; 53:731-6.

6. Barros MBA, Zanchetta LM, Moura EC, Malta DC. Auto-avaliação da saúde e fatores associados, Brasil, 2006. Rev Saúde Pública 2009; 43 Suppl 2:27-37.

7. Borim FSA, Barros MBA, Neri AL. Autoavaliação da saúde em idosos: pesquisa de base populacional no Município de Campinas, São Paulo, Brasil. Cad Saúde Pública 2012; 28:769-80.

8. Meireles AL. Auto-avaliação de saúde e bem estar dos adolescentes em um grande centro urbano: Estudo Saúde em Beagá [Masters Thesis]. Belo Horizonte: Faculdade de Medicina, Universidade Federal de Minas Gerais; 2010.

9. Jylhä M, Leskinen E, Alanen E, Leskinen AL, Heikkinen E. Self-rated health and associated factors among men of different ages. J Gerontol 1986; 41:710-7. 
10. French DJ, Browning C, Kendig H, Luszcz MA, Saito Y, Sargent-Cox K, et al. A simple measure with complex determinants: investigation of the correlates of self-rated health in older men and women from three continents. BMC Public Health 2012; 12:649.

11. McDaid O, Hanly MJ, Richardson K, Kee F, Kenny RA, Savva GM. The effect of multiple chronic conditions on self-rated health, disability and quality of life among the older populations of Northern Ireland and the Republic of Ireland: a comparison of two nationally representative cross-sectional surveys. BMJ Open 2013; 3:e002571.

12. Theme Filha MM, Szwarcwald CL, Souza Junior PRB. Medidas de morbidade referida e inter-relações com dimensões de saúde. Rev Saúde Pública 2008; 42:73-81.

13. Barros MBA, Cesar CLG, Carandina L, Torre GD. Desigualdades sociais na prevalência de doenças crônicas no Brasil, PNAD-2003. Ciênc Saúde Coletiva 2006; 11:911-26.

14. Santos SM, Chor D, Werneck GL, Coutinho ESF. Associação entre fatores contextuais e auto-avaliação de saúde: uma revisão sistemática de estudos multinível. Cad Saúde Pública 2007; 23:2533-54.

15. Caiaffa WT, Ferreira FR, Ferreira AD, Oliveira DLO, Camargos VP, Proietti FA. Saúde urbana: "a cidade é uma estranha senhora, que hoje sorri e amanhã te devora”. Ciênc Saúde Coletiva 2008; 13:1785-96.

16. Andrade ACS. Contexto social de moradia na prática de atividade física no lazer de residentes de um centro urbano: uma análise multinível, Estudo Saúde em Beagá [Masters Thesis]. Belo Horizonte: Centro de Pesquisas René Rachou, Fundação Oswaldo Cruz; 2013.

17. Friche AA, Diez-Roux AV, Cesar CC, Xavier CC, Proietti FA, Caiaffa WT. Assessing the psychometric and ecometric properties of neighborhood scales in developing countries: Saude em Beaga Study, Belo Horizonte, Brazil, 2008-2009. J Urban Health 2013; 90:246-61.

18. Cremonese C, Backes V, Olinto MTA, Dias-daCosta JS, Pattussi MP. Neighborhood sociodemographic and environmental contexts and self-rated health among Brazilian adults: a multilevel study. Cad Saúde Pública 2010; 26:2368-78.

19. Celio FA. Estudo Saúde em Beagá: fatores associados a autopercepção da extensão territorial da vizinhança [Masters Thesis]. Belo Horizonte: Faculdade de Medicina, Universidade Federal de Minas Gerais; 2013.

20. World Health Organization. Global recommendations on physical activity for health. Geneva: World Health Organization; 2010.

21. McDowell I, Newell C. Measuring health. A guide to rating scales and questionnaires. $3^{\text {rd }}$ Ed. New York: Oxford University Press; 1996.

22. Silva RA, Horta BL, Pontes LM, Faria AD, Souza LDM, Cruzeiro ALS, et al. Bem-estar psicológico e adolescência: fatores associados. Cad Saúde Pública $2007 ; 23: 1113-8$.
23. World Health Organization. Global strategy on diet, physical activity and health. Geneva: World Health Organization; 2000.

24. Lipschitz DA. Screening for nutritional status in the elderly. Prim Care 1994; 21:55-67.

25. Coutinho LMS, Scazufca M, Menezes PR. Métodos para estimar razão de prevalência em estudos de corte transversal. Rev Saúde Pública 2008; 42:992-8.

26. Victora CG, Huttly SR, Fuchs SC, Olinto MTA. The role of conceptual frameworks in epidemiological analysis: a hierarchical approach. Int J Epidemiol 1997; 26:224-7.

27. Camelo LV. Status social subjetivo, autoavaliação de saúde e tabagismo. Estudo Longitudinal de Saúde do Adulto (ELSA-Brasil) [Masters Thesis]. Belo Horizonte: Faculdade de Medicina, Universidade Federal de Minas Gerais; 2012.

28. Pagotto V, Bachion MM, Silveira EA. Autoavaliação da saúde por idosos brasileiros: revisão sistemática da literatura. Rev Panam Salud Pública 2013; 33:302-10.

29. Peres MA, Masiero AV, Longo GZ, Rocha GC, Matos IB, Najnie K, et al. Auto-avaliação da saúde em adultos no Sul do Brasil. Rev Saúde Pública 2010; 44:901-11.

30. Lee HY, Jang SN, Lee S, Cho S, Park EO. The relationship between social participation and self-rated health by sex and age: a cross-sectional survey. Int J Nurs Stud 2008; 45:1042-54.

31. Baum F, Bush R, Modra C, Murray C, Cox E, Alexander K, et al. Epidemiology of participation: an Australian community study. J Epidemiol Community Health 2000; 54:414-23.

32. Bjornstrom EES, Ralston ML, Kuhl DC. Social cohesion and self-rated health: the moderating effect of neighborhood physical disorder. Am J Community Psychol 2013; 52:302-12.

33. Stewart-Brown S, Layte R. Emotional health problems are the most important cause of disability in adults of working age: a study in the four counties of the old Oxford region. J Epidemiol Community Health 1997; 51:672-5.

34. World Health Organization. Global status report on noncommunicable diseases 2010. Geneva: World Health Organization; 2011.

35. Franks P, Gold MR, Fiscella K. Sociodemographics, self-rated health, and mortality in the US. Soc Sci Med 2003; 56:2505-14.

36. Lima-Costa MF, Peixoto SV, Firmo JOA. Validade da hipertensão arterial auto-referida e seus determinantes (projeto Bambuí). Rev Saúde Pública 2004; 38:637-42.

37. Duncan GJ, Raudenbush SW. Assessing the effects of context in studies of child and youth development. Educ Psychol 1999; 34:29-41.

Submitted on 14/May/2014

Final version resubmitted on 17/Jul/2014

Approved on 05/Sep/2014 